\title{
Need Assessment of Consultation Liaison Psychiatry amongst the Clinical Faculty
}

\author{
Rakesh K Chadda, Koushik Sinha Deb, Sathya Prakash, Mamta Sood \\ Department of Psychiatry \\ All India Institute of Medical Sciences, Ansari Nagar, New Delhi.
}

\begin{abstract}
Nearly $20-40 \%$ of patients with medico-surgical illnesses in general hospitals have a co morbid psychiatric illness or psychosocial issues, which interfere in improvement of the primary illness. It is important to assess the attitudes and awareness of non-psychiatrist clinicians about the co-existing psychiatric morbidity in their patients and their felt needs, which can help in mitigating this morbidity. The present study attempts to gauge the non-psychiatrist clinician's perception, felt needs and barriers to referral/ intervention in a tertiary care teaching hospital. A cross-sectional, descriptive, online questionnaire-based method was used. Of the 239 clinical faculty members, only 45 responded. Responses indicated that clinicians were aware of the existence and significance of psychological problems in their patients, but could do with further increased levels of awareness and more specific training in evaluation and intervention. Stigma, lack of awareness of available services, and lack of detailed understanding regarding psychological problems were the important barriers to referral/ intervention. Better teamwork, training and more manpower were the specific suggestions for improvement in the future.
\end{abstract}

Keywords: Psychosomatic medicine, stigma, general hospital psychiatry.

\section{Introduction}

Rapid changes in social structure and lifestyles have resulted in a surge in the prevalence of psychological conditions, particularly common mental disorders. Depression is now the second commonest cause of morbidity worldwide (1). In a general hospital setting, most of such patients initially present to medical and surgical departments, with only a small number presenting directly to psychiatry. Nearly $20-40 \%$ of patients attending various outpatients and in-patients clinical services for various medico-surgical problems also suffer from psychiatric illnesses like anxiety and stress-related disorders, depression, functional somatic syndromes, and substance use disorders or have psychosocial issues complicating their primary illness. Many of these remain unidentified and have a potential to increase physical morbidity, prolonging hospital stay and incurring increased cost to the system. Consultation liaison (CL) psychiatry is a speciality of psychiatry, in which a psychiatrist works in liaison with the primary clinical team and helps in diagnosis and management of patients with psychiatric problems in nonpsychiatric settings. It includes collaborative teaching and research activities with health professionals in non-psychiatric divisions (2). Taken together, CL psychiatry thereby provides the interface for collaborative management, 
training and research between psychiatry and other clinical departments and can, therefore, be of significant value in a general hospital setup. CL psychiatry derives its roots from psychosomatic medicine, a discipline concerned with the interplay of biological and psychosocial factors in the causation, course and outcome of various diseases (3).

Worldwide, although various models of CL services were developed in the 1970s and early 80s (4) evaluative research in training of CL psychiatry is currently lacking (5). In India too, though the first general hospital psychiatric units (GHPUs) was set up as early as 1933 and there may be more than 500 GHPUs in the country, there are very few CL psychiatry units in the country (6). The number of papers published on this topic from India are also few and leave much to be desired.

A survey in a major teaching hospital in Delhi found that $87 \%$ of physicians and surgeons were of the opinion that they would have been benefitted if their undergraduate training in psychiatry had been better (7). Similar findings were noted in another study involving 86 general practitioners and specialists (8). Several studies have found that timely referral and care of psychiatric problems in patients admitted in other wards improves quality of life and promotes early discharge. Alhuthail (9) demonstrated that nature of comorbid psychiatric diagnosis influenced the duration of stay in the ward. The referral time was noted as crucial and accounted for $22 \%$ variance of duration of ward stay. de Jonge et al (10) assessed the impact of psychiatric interventions on both the duration of ward stay as well as the quality of life of patients admitted in a medical ward. Psychiatric interventions were found to reduce duration of ward stay, particularly in the elderly. The quality of life was also enhanced by such interventions.

A recent study from Iran exploring the roadblocks in CL psychiatry reported that the most common reasons of physicians for not requesting psychiatric consultation were lack of time, access to psychiatrist and belief in the need for psychiatric consultation (11). A similar study from Saudi Arabia reported that poor psychiatric knowledge of medical doctors negatively influenced the referral rates to psychiatry and reflected the lack of integration of psychiatry and medicine at the training level (12). Poor psychiatric knowledge is also reflected in another study where majority of the medical specialists believed that the main task of the visiting psychiatrist was to advise them on psychosocial issues, while leaving clinical responsibility in their hands (13). Even in the Western countries like the UK, clinicians consider emotional assessment of routine patients impractical and referrals are avoided because of 'stigmatisation' (14). Underestimation of psychiatric morbidity by clinicians from different specialities is common and there is a general reluctance to refer patients to a psychiatrist for varied reasons (15).

A major problem for lack of integration of psychiatric services is considered to be the focus on brief consultations only in absence of liaison activities. Indeed, literature suggests that psychiatric liaison on medical wards produce a more positive attitude towards psychiatry and a higher consultation request rate. A study comparing $\mathrm{CL}$ as against consultation only model found that liaison activities were more favourably received by consultees than consultation alone and increased the consultation rate (16). Such liaison services have found more benefit in focussed departments like geriatrics and critical care medicine where delirium, dementia and psychiatric symptoms occur very frequently $(17,18)$, and also in oncology services where end of life care, depression and anxiety form a major focus of patients $(19,20)$. For most other clinical departments, specific conditions like suicidality, aggression and depression might be topics of focussed liaison discussions.

In India, we have very few CL psychiatry units. It is important to know the awareness, 
attitude and perceived needs of non-psychiatric clinicians about the need of CL psychiatry services in GHPUs. The present study was planned in this background to assess the perception of non-psychiatric clinical faculty regarding the nature and extent of mental health problems in their patients; to understand the factors influencing referral to a mental health specialist and, to assess their perceived needs for CL psychiatry services.

\section{Methods}

The Department of Psychiatry, All India Institute of Medical Sciences, New Delhi has started a dedicated CL psychiatry service in 2008. The service is provided by one senior resident, 2 junior residents on rotational posting, supervised by two faculty members. The study was conducted by the CL Psychiatry Team using a questionnaire-based survey approach with a cross sectional design.

For the purposes of this study, a semistructured 27 item questionnaire was developed by the authors. The contents of this questionnaire have been taken from literature review and clinical experience. The questionnaire was intended to be answered in a self-report fashion by the participants. The questionnaire collects non-identifying details such as subject of specialization, department of work, year of completing post-graduation and years of experience as faculty. The rest of the items in the questionnaire were intended to obtain data pertaining to the three broad areas of interest including the kind of psychiatric symptoms/illness seen by them in their clinical practice, reasons for seeking psychiatric referrals, their general awareness about psychiatric symptomatology, psychiatric medications and non-pharmacological treatments, and their awareness about CL psychiatry. The complete questionnaire can be obtained from the authors on request.

At the time of study, there were 239 faculty members working in various clinical disciplines excluding psychiatry. All clinical faculty members working currently at the institute $(n=239)$ were sent mail giving background of the study with an online version of the questionnaire created using the free platform of Google Documents and Google Survey. The faculty members were requested participation in the study. Two reminders were sent after one and three months. The questionnaire took about 10 minutes to fill and submit. Every participant was informed of the nature of the study and informed consent was obtained.

The study was approved by the Institute Ethics Committee. Full confidentiality was maintained. Individual characteristics of the respondents were tabulated and a content analysis of the responses was conducted.

\section{Results}

Responses were received from only 46 faculty members. Average experience of the participant faculty members from different disciplines varied from 14-22 years as faculty. The participant characteristics are given in Table 1. Thirty five respondents were not aware that CL Psychiatry was a separate sub-speciality with a designated workforce. Most of the participants saw about 10 inpatients and 50-100 outpatients per week. All but two of them agreed that focussed training of residents in this area would be useful.

\section{Content Analysis}

Most of the participants felt that their residents needed training to intervene for common psychiatric disturbances occurring in the context of the primary physical illness. A. comparable number of the respondents also highlighted the need for training on communication skills and building a better rapport with the patient.

Most participants felt that $20-40 \%$ of their patients seemed to have some psychological/ psychiatric difficulties. Of those with such psychological/ psychiatric difficulties, majority felt that upto $20 \%$ had an already diagnosed/ 
100 Rakesh K Chadda

Table 1: Characteristics of the participants $(N=46)$

\begin{tabular}{|l|c|c|}
\hline Department & Number of responses & $\begin{array}{l}\text { Average experience } \\
\text { as faculty in years }\end{array}$ \\
\hline Gastroenterology/ GI Surgery & 5 & 14.0 \\
\hline Paediatrics & 5 & 22.0 \\
\hline $\begin{array}{l}\text { Physical Medicine and } \\
\text { Rehabilitation }\end{array}$ & 4 & 17.5 \\
\hline Anaesthesia & 4 & 19.0 \\
\hline Community Medicine & 4 & 19.7 \\
\hline Deaddiction & 3 & 14.3 \\
\hline Cardiology/ Cardiac Surgery & 3 & 19.7 \\
\hline Otorhinolaryngology & 3 & 18.0 \\
\hline Others & 15 & - \\
\hline
\end{tabular}

diagnosable independent psychiatric illness and upto $40 \%$ may have problems seemingly secondary to the medical illness. An overwhelming majority also felt that upto one fifth of their patients may have a psychological problem masquerading as a physical problem. Anxiety was the most common symptom encountered, seen in almost half of their patients seen, while about a fifth of their patients had depressive symptoms. A smaller number of them reportedly may have had psychotic symptoms. About half of the participants thought that upto $20 \%$ of the patients had medically unexplained physical symptoms whereas about a third of the participants felt that the number may be as high as $40 \%$. Substance use problems and confusional states were also seen in less than $20 \%$ of the patient population.

Almost all participants agreed that psychological factors can influence course and outcome of physical conditions and their modification can expedite improvement. The basic evaluation and management of psychological problems were to be done by the primary treating doctor and also that such an evaluation was not being conducted. Opinion was divided on whether psychiatrists/ psychologists must be readily available for providing emotional support to patients for health-related anxiety. While a little over half of the participants did feel so, one third of them were either undecided or did not agree to the same. The respondents were undecided on whether time constraint was the reason for inadequate psychological evaluation with responses spread across the agree-disagree continuum, with a slight skew towards agreement. A little over half seemed to suggest that lack of awareness was a major reason. An overwhelming majority felt that psychotherapeutic as well as biological interventions were of great value in this profile of patients as well. Almost all agreed that their patients have been benefitted by psychiatric consultation in the past. Over one third of the participants were unsure whether common mental disorders in their patients should be managed by non-psychiatrist doctors themselves.

For anxiety and somatic symptoms, majority of the clinicians considered treating with an Selective Serotonin Reuptake Inhibitor (SSRI), benzodiazepine or reassurance/ supportive counselling and only a minority considered referral. For suicidal thoughts, disorganized behaviour, hallucinations, almost all participants considered referral. For depressive symptoms without suicidal thoughts, half of them considered starting an antidepressant on their own while the rest considered referral.

Three/fourth of the participants felt that despite referral, patients were reluctant and 
feared stigma. Two third felt that inclusion of a psychiatrist (like a senior resident) in the medical team would be of significant value.

Suggestions for the future included: training of all specialists in basic psychological evaluation, more manpower for CL services, combined clinics between psychiatry and other departments, better teamwork, and need for evaluation by senior/ more experienced psychiatrists.

Salient points of this content analysis are provided in Box 1.

\section{Discussion}

The present study attempted to understand not only the knowledge and attitudes of clinicians towards CL psychiatry services but also to understand barriers against adequate evaluation and referral, as well as, the felt needs of these clinicians. This is an improvement over many previous studies in India that have focussed only on knowledge and attitude.
Contrary to findings from certain previous studies, as well as popular belief, clinicians acknowledged the importance of psychiatric services, felt there was inadequate attention paid by them towards this, and showed eagerness towards improving the same. This is in keeping with an earlier study (14) that suggested that over the years, there have been significant changes for the better in attitudes of clinicians towards psychiatric services. Also in keeping with the findings of the same study was the fact that stigma was one of the main barriers for referral to be successful (14). Unlike many studies that seemed to suggest inadequate time as an important barrier, the present study did not endorse it so strongly (11). Also in contradiction with previous studies, most participants acknowledged that they were aware of the significant co-morbid presence of psychiatric/ psychological problems in their patients (13). In keeping with the idea of minimal undergraduate training, the study did find that there was considerable interest in clinicians to undergo basic training for psychological evaluation and management. Need for more mental health

\section{Box 1: Predominant themes emerging from content analysis}

\begin{tabular}{|l|l|}
\hline Theme & Predominant view \\
\hline Training needed for & $\begin{array}{l}\text { Evaluation/ intervention for anxiety/ depression } \\
\text { Communication skills } \\
\text { Good rapport }\end{array}$ \\
\hline Most common symptoms and their nature & $\begin{array}{l}\text { Anxiety, usually secondary to underlying } \\
\text { medical illness }\end{array}$ \\
\hline Reasons for failure of referral & $\begin{array}{l}\text { Lack of awareness } \\
\text { Stigma }\end{array}$ \\
\hline Nature of problem in referred individuals & $\begin{array}{l}\text { Suicidality } \\
\text { Psychotic symptoms }\end{array}$ \\
\hline $\begin{array}{l}\text { Nature of problems treated by clinicians } \\
\text { themselves }\end{array}$ & $\begin{array}{l}\text { Somatic symptoms } \\
\text { Anxiety } \\
\text { Depression without suicidality }\end{array}$ \\
\hline Suggestions for future & $\begin{array}{l}\text { Combined clinics, better teamwork } \\
\text { More of Liaison than just consultation } \\
\text { Training for other departments } \\
\text { More manpower and experienced personnel }\end{array}$ \\
\hline
\end{tabular}


specialists, a concern raised by previous studies were also echoed by the findings of the current study. The acknowledgement of the fact that treatment of psychological problems expedites improvement and discharge is a welcome change in the attitude of clinicians (14). In keeping with previous literature, CL psychiatry was preferred rather than just consultation-based ones (16). Besides the above, the current study also brought out several nuances of previously known facts. For instance, clinicians chose to treat anxiety and somatic symptoms on their own while choosing to refer patients with psychotic or suicidal ideas. It also demonstrated that the participants had a great deal of confidence in psychiatric interventions, both biological and non-biological. They also substantiated it citing prior good experience with referrals.

The present study had a small sample size. The response rate was very low and therefore it raises questions about the representativeness of the sample for the institute. Persons responding may be quite sensitive to psychological aspects creating a bias. The bias, if present, may explain the relatively more positive response pattern in comparison to previous studies. Additionally, the sample from a single centre may not be representative for the country. Future studies should include larger sample, multiple centres and more robust qualitative methodology like use of focus group discussions to get a more nuanced and deeper understanding of the situation.

\section{Conclusion}

The study found that clinicians were aware of the existence and significance of psychological problems in their patients, but could do with further increased levels of awareness and more specific training in evaluation and intervention. Stigma, lack of awareness of available services, and lack of detailed understanding regarding psychological problems were the important barriers to referral/ intervention. Better teamwork, training and more manpower were the specific suggestions for improvement in the future.

\section{References}

1. Ferrari JA, Charlson FJ, Norman RE, et al (2013). Burden of depressive disorders by country, sex, age, and year: findings from the Global Burden of Disease Study 2010. PLoS Med10(11): e1001547.

2. Lipowski ZJ (1983). Current trends in consultation-liaison psychiatry. Can $J$ Psychiatry 28:329-338.

3. Mayou R (2007). The development of general hospital psychiatry. In: Handbook of Liaison Psychiatry. Lloyd G, Guthrie E, eds. UK: Cambridge University Press.

4. Ali S, Ernst C, Pacheco M, Fricchione G (2006). Consultation-liaison psychiatry: How far have we come? Curr Psychiatry $\operatorname{Rep}$ 8:215-222.

5. Sollner W, Creed F (2007). European guidelines for training in consultation-liaison psychiatry and psychosomatics: report of the EACLPP Workgroup on Training in Consultation-Liaison Psychiatry and Psychosomatics. J Psychosom Res 62: 501-509.

6. Grover S (2011). State of consultationliaison psychiatry in India: current status and vision for future. Indian J Psychiatry 53:202-213.

7. Chadda RK, Shome S (1996). Psychiatric aspects of clinical practice in general hospitals: a survey of non-psychiatric clinicians. Indian J Psychiatry 38:86-93.

8. Gupta R, Narang RL (1987). Psychiatric training and its practice: a survey of 86 practitioners. Indian J Psychiatry 29:349352. 
9. Alhuthail YR (2008). Psychiatric consultations and length of hospital stay. Neurosciences 13: 161-164.

10. de Jonge P, Latour CH, Huyse FJ (2003). Implementing psychiatric interventions on a medical ward: effects on patients' quality of life and length of hospital stay. Psychosom Med 65: 997-1002.

11. Zarghami M, Farnia S, Khalilian AR, Amirian T (2014). Study of attitudes and practice of physicians regarding consultation-liaison psychiatry in teaching hospitals of Mazandaran, Iran. Iran J Psychiatry Behav Sci 8(2) : 38-43.

12. Alhamad AM, Al-Sawaf MH, Osman AA, Ibrahim IS (2006). Differential aspects of consultation-liaison psychiatry in a Saudi hospital. II: knowledge and attitudes of physicians and patients. East Mediterr Health J12:324-330.

13. Doron A, Ma'oz B, Fennig S, Weingarten MA, Mendlovic S (2003). Attitude of general practitioners towards psychiatric consultation in primary care clinic. Isr $J$ Psychiatry Relat Sci 40(2):90-95.

14. Morgan JF, Killoughery M (2003). Hospital doctors' management of psychological problems - Mayou \& Smith revisited. BrJPsychiatry 182:153-157.
15. Chadda RK (2001). Psychiatry in nonpsychiatric setting--a comparative study of physicians and surgeons. J Indian Med Assoc 99(1):24, 26-7, 62.

16. Schubert DS, Billowitz A, Gabinet L, Friedson W (1989). Effect of liaison psychiatry on attitudes toward psychiatry, rate of consultation, and psychosocial documentation. Gen Hosp Psychiatry 11(2):77-87.

17. Nogueira V, Lagarto L, Cerejeira J, Renca S, Firmino H (2013). Improving quality of care: focus on liaison old age psychiatry. Ment Health Fam Med 10(3):153-158.

18. Draper B (2000). The effectiveness of old age psychiatry services. Int $J$ Geriatr Psychiatry 15(8):687-703.

19. Olagunju AT, Aina OF, Fadipe B (2013). Screening for depression with Centre for Epidemiological Studies Depression Scale Revised and its implication for consultation-liaison psychiatry practice among cancer subjects: a perspective from a developing country. Psychooncology 22(8):1901-1906.

20. Chaturvedi SK (2012). Psychiatric oncology: Cancer in mind. Indian $J$ Psychiatry 54(2):111-118. 